\title{
Sputum induction for the diagnosis of pulmonary tuberculosis in infants and young children in an urban setting in South Africa
}

\author{
H J Zar, E Tannenbaum, P Apolles, P Roux, D Hanslo, G Hussey
}

\begin{abstract}
Background-Bacteriological confirmation of pulmonary tuberculosis is difficult in infants and young children. In adults and older children, sputum induction has been successfully used; this technique has not been tested in younger children.

Aims-To investigate whether sputum induction can be successfully performed in infants and young children and to determine the utility of induced sputum compared to gastric lavage (GL) for the diagnosis of pulmonary tuberculosis in HIV infected and uninfected children.

Subjects and methods-149 children (median age 9 months) admitted to hospital with acute pneumonia who were known to be HIV infected, suspected to have HIV infection, or required intensive care unit support. Sputum induction was performed on enrolment. Early morning GL was performed after a minimum four hour fast. Induced sputum and stomach contents were stained for acid fast bacilli and cultured for Mycobacterium tuberculosis. Results-Sputum induction was successfully performed in 142 of 149 children. $M$ tuberculosis, cultured in 16 children, grew from induced sputum in 15. GL, performed in 142 children, was positive in nine; in eight of these $M$ tuberculosis also grew from induced sputum. The difference between yields from induced sputum compared to GL was $4.3 \%(p=0.08)$. $M$ tuberculosis was cultured in 10 of $100 \mathrm{HIV}$ infected children compared to six of 42 HIV uninfected children $(p=0.46)$.

Conclusion-Sputum induction can be safely and effectively performed in infants and young children. Induced sputum provides a satisfactory and more convenient specimen for bacteriological confirmation of pulmonary tuberculosis in HIV infected and uninfected children.

(Arch Dis Child 2000;82:305-308)
\end{abstract}

Keywords: induced sputum; tuberculosis; HIV

Bacteriological confirmation of pulmonary tuberculosis in infants and children remains difficult. Sputum induction has been used to diagnose pulmonary tuberculosis in HIV infected and immunocompetent adults. ${ }^{1}$ Older children can produce or be induced to produce sputum; this method has been used for the diagnosis of Pneumocystis carinii pneumonia and more recently for tuberculosis. ${ }^{23}$ However, there are no reports of its use in infants or children younger than 3 years.

Gastric lavage (GL) is regarded as the standard procedure to obtain specimens for staining and culture of Mycobacterium tuberculosis in younger children because they swallow their sputum and do not expectorate. Studies comparing GL to induced sputum in adults with suspected tuberculosis reported induced sputum to be more effective ${ }^{45}$; however, one study reported that if GL was undertaken after sputum induction the procedures produced similar results. ${ }^{6}$ A study of 13 children with pulmonary tuberculosis reported an improved yield from GL when preceded by a nebulisation of superheated isotonic saline. ${ }^{7}$ There are no published studies comparing induced sputum to GL for culture of $M$ tuberculosis in children. Two studies of GL compared to bronchoalveolar lavage (BAL) in children suggest that GL provides a higher bacteriological yield than BAL for pulmonary tuberculosis. ${ }^{89}$

The aim of this study was to determine whether sputum induction can be successfully performed in infants and young children, to compare induced sputum with GL for the yield of $M$ tuberculosis in children with pulmonary tuberculosis, and to determine whether the yield was influenced by HIV status.

\section{Methods}

PATIENTS

A prospective one year study during 1998 was performed in the paediatric wards of four hospitals in Cape Town, South Africa-Red Cross War Memorial Children's Hospital, Somerset, Conradie, and Groote Schuur. Children enrolled in this study were participants in a larger study to determine the aetiology of acute pneumonia in hospitalised, HIV infected children. Children with a primary diagnosis of pneumonia according to World Health Organisation criteria $^{10}$ (defined as the presence of tachypnoea or lower chest indrawing) and who were known to be HIV infected, were suspected of having HIV infection, or were admitted to the intensive care unit (ICU) but were not intubated were studied. A suspicion of HIV infection was based on the presence (in addition to pneumonia) of two or more of the following: generalised lymphadenopathy, weight below the $3 \mathrm{rd}$ centile for age, hepatomegaly, splenomegaly, oral candidiasis, enlarged parotid glands, or chronic diarrhoea. Children were enrolled during working hours from Monday to Friday. Informed consent for enrolment in the study and for HIV testing (in 
Table 1 Characteristics of children in whom sputum induction was performed

\begin{tabular}{lll}
\hline Characteristic & $\begin{array}{l}\text { Children without } \\
\text { TB }(n=126)\end{array}$ & $\begin{array}{l}\text { Children with } \\
\text { TB }(n=16)\end{array}$ \\
\hline Age (months) & $9(3-21.5)$ & $12(7-25)$ \\
Male:female & 1.2 & 2.2 \\
ICU admission, $\mathrm{n}(\%)$ & $18(14)$ & $2(12)$ \\
HIV positive, $\mathrm{n}(\%)$ & $90(71)$ & $10(63)$ \\
TB contact, $\mathrm{n}(\%)$ & $24(19)$ & $7(44)^{\star}$ \\
Use of supplemental $\mathrm{O}_{2}, \mathrm{n}(\%)$ & $83(66)$ & $8(50)$ \\
Baseline $\mathrm{O}_{2}$ saturation in air & $94(90.5-97)$ & $93.5(88-98)$ \\
Baseline $\mathrm{RR}$ & $50(40-60)$ & $53(40-63.5)$ \\
\hline Continuous variables expressed as median & $(25$ th to 75 th \\
percentile). & \\
TB, tuberculosis; ICU, intensive care unit; RR, respiratory rate. \\
${ }^{\star} \mathrm{p}=0.02$.
\end{tabular}

children with suspected HIV infection or admitted to ICU) was obtained from a parent. The study was approved by the Research and Ethics Committee of the University of Cape Town.

A history and physical examination were performed in every child enrolled. HIV infection was confirmed by two positive enzyme linked immunosorbent assay (ELISA) tests (Vironostika HIV Uniform II, Organon Teknika, Holland) in children older than 18 months or by a positive ELISA and polymerase chain reaction (PCR; Amplicor HIV-1, Roche Diagnostic Systems) in younger children. Tuberculosis was diagnosed only when cultures of induced sputum or GL grew $M$ tuberculosis.

GASTRIC LAVAGE

Early morning GL was performed in children younger than 5 years old after an overnight fast of at least four hours. A nasogastric tube was passed before the child arose and the gastric contents aspirated. Normal saline $20 \mathrm{ml}$ was inserted down the tube, left for two to three minutes, and then aspirated. Additional 5-10 $\mathrm{ml}$ normal saline aliquots were inserted and aspirated until a minimum of $20 \mathrm{ml}$ of aspirate was obtained. Gastric aspirates were placed in a sterile, sodium carbonate containing tube and taken to the laboratory. GL was ideally performed on two or three consecutive mornings; however, repeated lavages were not possible in many children owing to other factors such as discharge from hospital or subsequent intubation.

\section{SPUTUM INDUCTION}

Sputum induction was undertaken on the day of enrolment after a two to three hour fast. A physiotherapist or research nurse trained in the use of this technique performed the procedure. Children were pretreated with $200 \mu \mathrm{g}$ salbutamol given via metered dose inhaler with attached spacer (Babyhaler, GlaxoWellcome) to prevent the occurrence of bronchoconstriction. ${ }^{11}$ A jet nebuliser (GRS, Intersurgical, UK) attached to oxygen at a flow rate of $5 \mathrm{l} / \mathrm{min}$ delivered $5 \mathrm{ml}$ of $5 \%$ sterile saline for 15 minutes. Thereafter physiotherapy techniques including chest percussion, vibration, shaking, and active cycle breathing were applied. Sputum was obtained either by expectoration (in children able to cooperate) or by suctioning through the nasopharynx or oropharynx using a sterile, mucus extractor of catheter size 6 . Specimens were transported directly to the laboratory for processing.

\section{LABORATORY METHODS}

GL specimens collected on consecutive days were pooled before culturing, while induced sputum samples were cultured singly. Specimens were decontaminated with sodium hydroxide, and after neutralisation with buffered saline were concentrated by centrifugation. The resuspended deposit was used to make a smear for microscopy. Prepared smears from the concentrated samples were stained with Auramine-O and examined by fluorescence microscopy for the presence of acid fast bacilli. A Bactec 12B bottle (Becton Dickinson, USA) containing supplemented Middlebrook medium was inoculated with $0.5 \mathrm{ml}$ of sample, incubated at $37^{\circ} \mathrm{C}$, and monitored for growth twice weekly for the first two weeks, then weekly for a total incubation period of six weeks. Positive mycobacterial growth was confirmed by a stain for acid fast bacilli as well as by PCR using primers prepared in house.

STATISTICAL ANALYSIS

Results were analysed using Epi6.04 (CDC, Atlanta, USA). Comparison between HIV infected and uninfected children was made using the $\chi^{2}$ and Kruskal-Wallis tests where appropriate. The yield of $M$ tuberculosis from induced sputum and gastric lavage was compared using the McNemar test.

\section{Results}

Sputum induction was successfully performed in 142 of 149 children with a median (25th to 75 th centile) age of 9 (3-20) months (table 1). The youngest child in whom sputum was successfully obtained was 1 month old. Seven children were either considered too ill to tolerate sputum induction or developed increasing tachypnoea or cough during nebulisation or suctioning, necessitating termination of the procedure. Only a minority of children (10\%) could expectorate and sputum was obtained by suctioning in the remainder. No serious adverse reactions occurred during sputum induction but there were minor events including mild epistaxis in six, increase in coughing in eight, and wheezing responsive to an inhaled bronchodilator in three. The baseline median respiratory rate of children was 50 (40-60) and arterial oxygen saturation in air was $94 \%$ (92$97 \%$ ). One hundred children were found to be HIV infected.

$M$ tuberculosis was cultured from sputum or GL in 16 children. Sputum cultures grew $M$ tuberculosis in 15 of 142 children but GL was positive in only nine (table 2). A single GL sample was obtained in 39 children (of which two were positive); there were two pooled lavage specimens in 77 (four positive), and three pooled specimens in 26 (three positive). In only one case was the GL culture positive for mycobacteria while the corresponding sputum was negative. The difference $(95 \%$ confidence interval) between yields for $M$ tuberculosis from culture of induced sputum compared to GL 
Table 2 Diagnosis of children with tuberculosis by sputum induction or gastric lavage

\begin{tabular}{lll}
\hline & \multicolumn{2}{l}{ Gastric lavage } \\
\cline { 2 - 3 } Induced sputa & $\begin{array}{l}\text { Culture positive } \\
(n)\end{array}$ & $\begin{array}{l}\text { Culture negative } \\
(n)\end{array}$ \\
\hline Culture positive $(n)$ & 8 & 7 \\
Culture negative ( $n)$ & 1 & 126 \\
\hline
\end{tabular}

specimen was $4.3 \%$ ( $0 \%$ to $5.6 \%$; $\mathrm{p}=0.08)$. Of the specimens culture positive for $M$ tuberculosis, three of 15 induced sputum (20\%) and three of nine GL specimens (33\%) were positive by microscopy for acid fast bacilli.

The median age of children with bacteriological confirmation of tuberculosis was 12 (7-25) months which was similar to those without tuberculosis (9 (3-21.5) months; $\mathrm{p}=0.19)$. The youngest child in whom $M$ tuberculosis was cultured from induced sputum was 3 months of age while seven of 15 children were less than 1 year. No child had cavitatory tuberculosis. Ten children culture positive for $M$ tuberculosis were HIV infected. This represents $10 \%$ of all HIV infected children, which is similar to the percentage of HIV negative children with tuberculosis (six of 42, 14.3\%) (odds ratio $0.67(0.2-2.41), \mathrm{p}=0.46)$.

\section{Discussion}

This study showed that sputum induction can be effectively and safely performed in infants and young children including those with HIV infection. The procedure could be successfully performed in infants as young as 1 month of age. Sputum induction was well tolerated even in children who were hypoxic or who had AIDS. Although we were unable to perform continuous monitoring of arterial oxygen saturation during sputum induction, very few children were unable to complete the procedure and no child subsequently appeared to deteriorate clinically. Sputum induction was a more sensitive method than GL for culture of $M$ tuberculosis, detecting almost twice the number of children with pulmonary tuberculosis. The lack of statistically significant difference between the yield from induced sputum compared to GL is most likely a result of the small number of children with tuberculosis and a larger study would be useful. The small number of tuberculosis cases reflects the relatively low risk of infection among the study group as they were hospitalised for acute pneumonia. Nevertheless, pulmonary tuberculosis was a common diagnosis in this group of children from a high HIV prevalence population who had clinical features of acute respiratory infection. Further study of the efficacy of sputum induction in children presenting with chronic illness suggestive of tuberculosis, such as failure to thrive and persistent fever, is warranted.

Childhood tuberculosis is an increasingly important public health problem, particularly in developing countries. The diagnosis of tuberculosis is notoriously difficult in children, especially those who are HIV infected, owing to the development of anergy which limits the use and importance of the skin tuberculin test. In addition, other HIV associated lung diseases may mimic the clinical and radiological picture of pulmonary tuberculosis. Gastric aspirates have been reported to give isolation rates of $M$ tuberculosis ranging from $28 \%$ to $40 \%$ in children with suspected tuberculosis, although higher rates of up to $75 \%$ have been described in infants. ${ }^{12-14}$ Although GL was performed according to a standard protocol, different nurses performed this procedure as an early morning preprandial specimen was required. The relatively lower culture rate of $M$ tuberculosis from GL compared to sputum induction may therefore reflect some variability in GL technique and the lack of well standardised guidelines for the procedure.

Sputum induction mobilises secretions from the lower respiratory tract. Hypertonic saline, deposited in the lower airways, causes interstitial fluid to move into this area by osmosis. ${ }^{15}$ Furthermore, hypertonic saline stimulates the cough reflex, causing secretions from the lower respiratory tract to be moved upwards. The efficacy of sputum induction in this study may be a result of the application of a standard technique by a few people trained in its use, and to rapid processing of the specimen by the laboratory. Nevertheless, this technique is relatively simple, does not require sophisticated equipment, and can be taught to health care workers. Care should be taken to ensure that sputum induction does not facilitate the spread of tuberculosis to other patients or staff. Sputum induction in children is unlikely to result in tuberculosis transmission owing to the paucibacillary nature of their disease as evidenced by a minority of children who were smear positive. Nevertheless, it is recommended that sputum induction be performed in a room with negative pressure ventilation ${ }^{16}$; in the absence of such a facility (as occurs in most developing countries), the procedure should be done in a well ventilated room and equipment sterilised between patients. Whereas GL is time consuming, distressing to the child and care giver, and should be repeated on consecutive days, induced sputum is easier to perform, relatively non-invasive, does not require hospitalisation, and can be repeated if necessary. Although GL can be successfully performed in an ambulatory setting, ${ }^{17}$ the majority of children require hospitalisation; in contrast sputum induction is easily done as an outpatient procedure.

In conclusion, sputum induction can be effectively performed and is well tolerated and safe even in infants. Induced sputum is better than GL for the isolation of $M$ tuberculosis in both HIV infected and uninfected infants and children. The bacteriological yield from sputum or GL for pulmonary tuberculosis does not differ by HIV status. Use of induced sputum should be considered as a first line investigation in children suspected of having pulmonary tuberculosis, especially in circumstances in which a culture confirmed diagnosis should be vigorously sought (such as when the source case is unknown, drug resistance is suspected, or cutaneous anergy occurs). 
This work was funded by the MRC, South Africa, an ASTRA Respiratory Fellowship (HZ) awarded by the South African Pulmonology Society, and the ICH Fund, Red Cross Children's Hospital. We thank the laboratory staff, interpreters, pharmacists, social workers, ward doctors, nursing staff, and Ms W Isaacs for help with administering the study. We acknowledge Dr George Swingler for advice on statistics and review of the manuscript. Finally, we thank the children and their parents for participating in the study.

1 Parry CM, Kamoto O, Harries AD, et al. The use of sputum induction for establishing a diagnosis in patients with suspected pulmonary tuberculosis in Malawi. Tuber Lung Dis 1995;76:72-6.

2 Ognibene FP, Gill VJ, Pizzo PA, et al. Induced sputum to diagnose Pneumocystis carinii pneumonia in immunosuppressed pediatric patients. F Pediatr 1989;115:430-3.

3 Shata AMA, Coulter JBS, Parry CM, et al. Sputum induction for the diagnosis of tuberculosis. Arch Dis Child 1996;74:535-7.

4 Jones FL. The relative efficacy of spontaneous sputa, aerosol-induced sputa and gastric aspirates in the bacteriologic diagnosis of pulmonary tuberculosis. Dis Chest 1966; 50:403-8.

5 Lillehei JP. Sputum induction with heated aerosol inhalations for the diagnosis of tuberculosis. Am Rev Respir Dis $1961 ; 84: 276-8$

6 Carr DT, Karlson AG, Stilwell GG. A comparison of cultures of induced sputum and gastric washings in the diagnosis of tuberculosis. Mayo Clin Proc 1967;42:23-5.

7 Giammona ST, Zelkowitz PS. Superheated nebulized saline and gastric lavage to obtain bacterial cultures in primary pulmonary tuberculosis in children. Am $\mathcal{F}$ Dis Child 1969;117:198-200.
8 Abadco DL, Steiner P. Gastric lavage is better than bronchoalveolar lavage for isolation of Mycobacterium tuberculosis in childhood pulmonary tuberculosis. Pediatr Infect Dis f 1992;11:735-8.

9 Somu N, Swaminathan S, Paramasivan CN, et al. Value of bronchoalveolar lavage and gastric lavage in the diagnosis of pulmonary tuberculosis in children. Tuber Lung Dis 1995;76:295-9.

10 World Health Organisation Programme for the Control of Acute Respiratory Infections. ARI programme management. A training course. Module on National Policies. Geneva: World Health Organisation, 1990

11 Belcher NG, Lee TH, Rees PJ. Airway responses to hypertonic saline, exercise and histamine challenges in bronchial asthma. Eur Respir 7 1989;2:44-8.

12 Lloyd AVC. Bacteriological diagnosis of tuberculosis in children. A comparative study of gastric lavage and laryngeal swab methods. East Afr Med $\mathcal{f} 1968 ; 45: 140-3$.

13 Starke JR, Yaylor-Watts KT. Tuberculosis in the pediatric population of Houston, Texas. Pediatrics 1989;84:28-35.

14 Vallejo JG, Ong LT, Starke JR. Clinical features, diagnosis and treatment of tuberculosis in infants. Pediatrics 1994;84: $1-7$.

15 O'Byrne P, Hargreave F. Non-invasive monitoring of airway inflammation. Am f Respir Crit Care Med 1994;150:S100-2.

16 Centres for Disease Control. Guidelines for preventing the transmission of tuberculosis in health-care settings with special focus on HIV-related issues. MMWR Morb Mortal Wkly Rep 1990;39:1-29.

17 Lobato MN, Loeffler AM, Furst K, Cole B, Hopewell PC. Detection of Mycobacterium tuberculosis in gastric aspirates collected from children: hospitalization is not necessary. Pediatrics 1998:102:e40. 